\title{
PROCEDURES AND MECHATRONIC DEVICE FOR THE PREPARATION OF CHEMICAL CONTAMINATED SAMPLES „ON SITE"
}

\author{
Nicoleta Petrea ${ }^{1}$, Răzvan Petre ${ }^{2}$, Neculai Dorin Oancea ${ }^{3}$, Vasile Somoghi ${ }^{4}$, Alexandru Marin $^{5}$, Dana Ciutac ${ }^{6}$ \\ ${ }_{1,2}$ Scientific Research Centre for CBRN Defense and Ecology, \\ 225 Olteniței Road, District 4, 041309, Bucharest, Romania \\ 3,4,6SC ATLAS CORP SRL, \\ 31 Lt. Scarlat Mareș Street, District 3, 031875, Bucharest, Romania \\ ${ }^{5}$ University POLITEHNICA of Bucharest \\ 313 Splaiul Independentei, District 6, 060042, Bucharest, Romania \\ E-mail: nicoleta.petrea@yahoo.com; vsomoghi@yahoo.com
}

\begin{abstract}
This paper presents the realization and testing of an automatic system prototype, with civil and military applications in the fields of security and defence, which can be used on site for testing potentially chemically contaminated samples. This equipment is designed under the principles of an integrated mechatronic device and is particularly useful for the intervention teams that have to respond to chemical and ecological incidents (accidents or terrorist attacks), as well as to the decision factors because it provides them, in a very short time, the necessary information to take urgent and informed action for the neutralization of the local factors generating the crisis (chemical war agents, toxic industrial compounds, explosives, drugs, etc.), for the protection of the personnel and the environment, for choosing the appropriate decontamination means and medical treatment. $[1,2,3,4]$. Taking into account the fact that in this type of interventions 'time' is very important, this system is necessary. The mechatronic equipment has been designed to process, in a short time, all kinds of samples (solid, liquid, gases or complex substances' amalgams), integrating a number of preparation techniques: SPE (Solid Phase Extraction), SPME (Solid Phase Microextraction), Head Space, adsorption on TENAX adsorbent resin tubes etc. for the sample preparation on the spot. As a result, time and resource consuming activities are spared: sample collection, packing and labelling, transport to a specialized lab, classical testing in the lab. [5] From a scientific point of view, the use of SPE, SPME, Head Space techniques in the collection and processing of samples for the identification of organic pollutants does not represent an innovation, being successfully used for over 20 years. The innovation factor is brought by the utilization of this techniques for the collection and processing of samples contaminated with highly dangerous compounds from CWAs (chemical warfare agents) class in field situations, in an automatic manner. From a technological point of view, the main innovation is comprised of the integration of these separate techniques in an unitary mechatronic system which would comprise, besides extraction cartridges with different adsorbents, a collection system with an automatic pump, electronical control gadgets, sample heating gadget, time measuring gadget. Our mechatronic equipment comes in handy in the detection and identification of interest compounds, because the existent detection equipment can only identify substances in gas form, leaving the risk of not detecting the dangerous substances with low volatility. [6] Also, in the case of instrumental mechatronic techniques of analysis and identification of the compounds of interest the previous collection of samples is necessary in order to bring them in a state compatible with the analytic instrument, laborious process, that takes a lot of time and reagents in the case "classical methods of process in lab".
\end{abstract}

Keywords: Completely automated portable mechatronic device, rapid samples processing, on site, chemical contamination, detection and identification of the toxic compounds.

\section{Introduction}

The detection and identification of local hazard generating factors, especially chemical war agents and industrial toxic substances is the first step in the neutralization process. Without the exact identification of the chemical agent that was used and of its physical-chemical properties, you cannot talk about the staff's safety, intervention or medical help. Because of this reason, the detection and 
identification of this compounds was and remains a priority in the defence against chemical, biological and radiological threat. $[1,2,4,7]$

In the past few years, techniques and technologies that follow the realization of detection in the shortest time, at the same time as the accurate identification of the used agent, have been developed. Even though there have been notable results, a series of factors slows down the detection and identification process. Out of these factors, two remain decisive:

- the first one relates to the assurance of the compatibility between the instrumental technique that was used and the physical-chemical properties of the agent that will be identified;

- the second factor refers to the presence in real mediums of complex substances' amalgams, issue that affects the detection process, up to its failure.

The detection and identification of the substances that can be factors in generating crisis (CWAs, dangerous substances, explosives and drugs) has been a major preoccupation of the research institute, notable results being obtained especially starting with the 80s. The application on a large scale of IMS technology, for detecting interest substances, led to the realisation of specialized devices, with a great accuracy, portable and user-friendly (IMS, Raid M, Rapid, AP2C, AP4C, CAM, etc.). As a result, these devices led to the detection of the full range of substances of interest. At the same time, the perfecting of the spectrometric technique, known since the $60 \mathrm{~s}$, is carried on and has led to the making of the transportable analytic instruments (GC/MS, FTIR, NMR, IR, etc.) capable of analysing in hard field conditions, for the precise and performant identification of interest substances.

Due to the fact that in the past years this category of equipment has been used in bad conditions (conflictual situations, drills, applications, etc.) their limitations came to light. Thus, it has been shown that this instruments can only detect substances in vapour state and in relatively "clean" environments (natural environments).[8] In dense urban environments and highly populated areas, where the air contains a full range of substances (smoke, hydrocarbs, pesticides, exhaust gases, burn resulted gases emitted by the industrial chimneys, cosmetic products etc.), many of this dispositives producing false alarms (either starting an alarm in the absence of the interest compound, or, even though the interest compound is present, them failing in detecting it).

Because of this reason, the main objective of the production of this devices is the development of a product highly resistant to interference. Meanwhile, there are interest compounds which are not in a gas state or are dissolved in liquid or solid mixtures, case in which detection errors might occur. Consequently, there are researches for the production of systems which volatilize the compounds in the matrix for the facilitation of substances' detection.

As for the identification, GC/MS transportable systems can analyse gas samples collected on an adsorption system (different types of adsorbent resins), but with restrictions for the liquid and solid samples. This impediments are overcome in labs by a series of steps, comprised in a faze called the sample's preparation. Even though it is laborious and takes a lot of time and a high quantity consumption of reagents, the substance's preparation is easily done in specialized laboratories. But, it becomes difficult in field conditions.

The large companies that produce performant chemical detection and identification devices (Smith Detection, Brucker Daltonics etc.) that became part of the military, fire fighter, intervention troupes and security equipment including the Army and the Internal Affairs Ministry of Romania, are interested in the development and perfecting of on site collection and preparation devices. The performance, the high time consumption, the high costs of detection and identification equipments require the development of sampling and sample preparation systems for the rapid delivery of the samples in a form compatible with the analytical instruments rather than the development of new detection and identification systems that do not solve the problem of detection and identification at all times.

In Romania, the research on detection and identification equipment has been stuck since the 80 s. Due to this, the throwback of 30 years will be hard to overcome.

Consequently, we have decided to design a unitary system, auxiliary to the chemical detection and identification devices, that would facilitate a fast detection and identification of interest compounds in whichever state of aggregation and in any kind of mixture: substances with high persistence and low volatility that cannot be detected by existent devices with low detection span, fairly high and dangerous, at the same time (HD, HN, L , Vx, etc.), explosives and drugs that have a low vapour tension, fact that makes it harder and limits their detection. As a follow up on the research we have developed a completely automatic portable device that can process samples of any kind, on the spot, in a very short time and with a reduced reagents and manpower consumption.

\section{Destination}

The sample processing device, designed under the principles of an integrated mechatronic equipment, is meant for the CBRN research. It can be used both in lab conditions (230V plug in) and in field conditions (intervention car's battery plug in or its own rechargeable battery $12 \mathrm{~V}$ ), being able to be 
utilized by only one operator dressed in adequate equipment for the particular hazard situation (CBRN costume, gas mask, gloves, etc.).

It is a portable system that assures the processing of solid and/or liquid samples, for the facilitation of the detection of hazard substances, in the case of the use of IMS detection equipments (ionic mobility spectrometry), flame photometric detectors and the indicative tubes and the process of identification of this substances, in the case of the use of identification equipment based on GC/MS technologies.

\section{Composition}

The sample processing mechatronic device is put into a hard plastic cover (ABS High Impact). We have decided on a "troller"-like cover, which would ease the operator's effort and which facilitate the transport on site. In the in the case's over lid there would be made spaces for auxiliary components and on site necessary accessories.

It is equipped with a battery power supply, which ensures work autonomy even in the most inaccessible places, where the intevention car can not reach.

The electrical and mechanical components, part of the mechatronic device, are assembled in different cases, made out of polypropylene, for the prevention of the destruction of any electric components in the case of any reagents leakage, and the only use components (testing paper, extraction cartridge, etc.) are positioned so that the operator can easily access and change them.

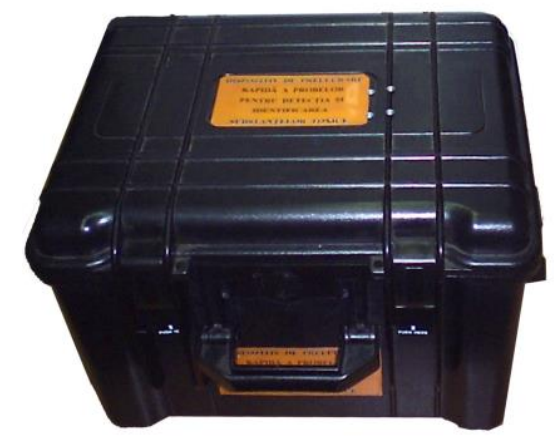

Figure 1: Device for preparation of chemical contaminated samples.

It is made out of two modules, the command and control unit, automatization soft and auxiliary systems [6].

I. Sample conditioning module, which has two functions:

- the processing of liquid and solid samples for the detection of toxic compounds;
- the processing of solid samples for the identification of adsorbed toxic compounds in the adsorbent resin tubes.

The Sample conditioning module consists of: heating mantle, electrical resistance for heating, temperature sensor, sample conditioning enclosure, micropump, TENAX adsorbent resin tube, connector for detector connection, hose, quick coupler, etc.

The sample heating system and the micropump that assures the transfer of the volatile compounds of interest receive commands through a microcontroller, the working algorithm being chosen and managed by the operator through a touchscreen.

II. Liquid sample processing module, configured for the two types of samples:

-Aqueous sample processing system, based on the C18 cartridge extraction principle.

It consists of: 3 chemical reagent vessels (methanol, water, methylene chloride), reagents dosing system, extraction cartridge C18.

-Organic solvent sample processing system, based on the Si cartridge extraction principle.

It consists of: 2 chemical reagent vessels (hexane, acetone), reagents dosing system, $\mathrm{Si}$ extraction cartridge.

The reagents dosing systems ensure the pumping of rigorously controlled quantities of reagents into the working chamber, the pumping elements being powered by stepper motors that assure an exact race. The transfer of the reagents and liquids samples is directed through hydraulic minicircuits branched out with the help of solenoid valves and transfer micropumps. All the active elements (stepby-step engines, solenoid valves, micropumps) receive commands through the microcontroller, having a precise control of the working steps, the reagents quantities and the timings necessary for the correct processing of the samples. The system interface to the user consists of a touch screen.

The auxiliary equipment comprises of: rooms for sample conditioning, TENAX adsorbent resin tubes; adaptors for the connection to the detector; C18 extraction cartridge; Si extraction cartridges; vials for liquid samples; syringes for liquid sample collection; utensils for solid sample collection (spatula, tweezers, cutter); reagent containers; electrical cord; protection equipment.

The configuration of the system makes the transport and service way easier. The system can be prepared for the sample's processing in less than 5 minutes. When turned on or in case of any functioning deficiency, the device is programmed to send acoustic and visual signs. 
The operator choses from the screen the working mode of the system, taking into account the necessities and demands, as followed:

- for the detection of toxic compounds with low volatility press "Samples conditioning program", that commands the start of the sample conditioning mode, ready for detection steps (the sample conditioning room with the "nose" installed in the device)

- for the identification of toxic compounds from aqueous samples press "Aqueous samples program", that commands the start of the submodule of aqueous samples processing;

- for the identification of toxic compounds from organic solvent based samples press "Organic samples program", that commands the start of the submodule of organic solvent samples processing;

- for the identification of toxic compounds from solid samples press "Samples conditioning program", that commands the start of the sample conditioning module, ready for the identification step (the sample's conditioning room has an adapter for the adsorbent tubes and adsorbent tubes installed in the device).

\section{Functional Testing}

While the system started taking shape, for its functionality check, there took place tests in laboratory conditions [9].

For the sample conditioning mechatronic model's testing for the detection of toxic compounds they have been contaminated with substances of interest (HD), in known concentrations, solid and liquid samples. The contaminated samples have been collected and processed with the mechatronic equipment and for its functionality testing an ACR and toxic industrial substances detection device, based on IMS spectrometry, has been used,. It is considered that the module worked correct if the IMS spectrometer detects the substance with which the sample was contaminated.

For the sample conditioning mechatronic module's testing for identifing toxic compounds there have been contaminated with substances of interest (HD), in known concentrations, soil samples and other known solid substances. The samples have been collected and processed with the mechatronic equipment and for checking its functionallity there has been used a gas chromatograph coupled to a mass spectrometer (GC/MS) system. It is considered that the module worked correctly if after the GC/MS analysis there took place the HD identification.

For the testing of the processing mechatronic module for liquid samples they have been contaminated with interest substances (DMMP dimethyl methylphosphonate), in known concentrations, water based and organinc samples. The constaminated samples have been collected and processed with the equipment and for checking its functionallity there has been used a GC/MS system. It is considered that the module worked correctly if after the GC/MS analysis there took place the DMMP identification.

Caution: The manipulation of any type of CWAs might cause severe injuries and/or death and is strictly regulated under CWC (Chemical Weapons Conventions) agreements and its production, storage and use require special authorization. Therefore, all reactions were performed by trained personal using safety procedures in a closed system or in a hood under good ventilation.

Table 1. Functional testing results

\begin{tabular}{|l|c|c|}
\hline \multicolumn{1}{|c|}{ Test } & $\begin{array}{c}\text { Admitted value (according to } \\
\text { the development } \\
\text { specification) }\end{array}$ & Resulted value \\
\hline $\begin{array}{l}\text { Conditioning capacity of solid and liquid } \\
\text { samples for the detection of toxic } \\
\text { substances with low volatility (HD), by IMS } \\
\text { spectrometry }\end{array}$ & $\begin{array}{c}\text { Detection of HD in the } \\
\text { processed samples, by IMS } \\
\text { spectometry }\end{array}$ & $\begin{array}{c}\text { In the processed samples } \\
\text { there has been identified } \\
\text { HD, by using an IMS } \\
\text { spectometer }\end{array}$ \\
\hline $\begin{array}{l}\text { Conditioning capacity of soil and other } \\
\text { known solid materials samples for the } \\
\text { identification with GC/MS of toxic } \\
\text { substances with low volatility (HD) }\end{array}$ & $\begin{array}{c}\text { Identification of HD in the } \\
\text { processed samples, by GC/MS } \\
\text { analysis, in the concentration } \\
\text { of } 0.02 \text { ppm }\end{array}$ & $\begin{array}{c}\text { In the processed samples } \\
\text { there has been identified } \\
\text { HD, by using a GC/MS } \\
\text { analysis }\end{array}$ \\
\hline $\begin{array}{l}\text { The capacity of processing water based } \\
\text { samples for the identification of toxic } \\
\text { substances (DMMP) by GC/MS }\end{array}$ & $\begin{array}{c}\text { Identification of DMMP in } \\
\text { anocessed samples, by GC/MS } \\
\text { analysis, in the concentration } \\
\text { limit of } 10^{-3}-10^{-4} \mathrm{mg} / \mathrm{l}\end{array}$ & $\begin{array}{c}\text { In the processed samples } \\
\text { there has been identified } \\
\text { DMMP, by GC/MS analysis }\end{array}$ \\
\hline $\begin{array}{l}\text { The capacity of processing organic solvent } \\
\text { samples for the identification of toxic } \\
\text { substances (DMMP) by GC/MS }\end{array}$ & $\begin{array}{l}\text { Identification of DMMP in } \\
\text { processed samples, by GC/MS } \\
\text { analysis, in the concentration } \\
\text { limit of } 10^{-3}-10^{-4} \mathrm{mg} / \mathrm{l}\end{array}$ & $\begin{array}{c}\text { In the processed samples } \\
\text { there has been identified } \\
\text { DMMP, by GC/MS analysis }\end{array}$ \\
\hline
\end{tabular}


The solid and liquid rezidual substances will be collected and decontaminated according to the working instructions and valid procedures.

\section{Conclusions}

The main purpose for which this mechatronic product was created is the fast processing of liquid or solid samples on site, for the detection and identification of chemical toxic agents. It can be used in labs and in the field, having its own power bank $(12 \mathrm{~V})$. It can be connected at the a main electrical source $(230 \mathrm{~V})$ as well as a car's battery (12V).

The compact constructive shape, the reduced volume, the clear inscriptioning, the user-friendly turn on function and utilization conferes the product a very high operativity level. Because of the troller like case, it can be easily moved even in hardly accessible places, where the intevention car cannot drive in.

The mechatronic product permits not only the identifiction of toxic agents found in reduced quantities in different types of matrices, but also the reduction of the time needed for the processing of this type of sample.
The time needed for the start of the work regimen is low (aprox. 4 minutes), which allowes a fast determination. In real conditions, the time affected by the preparation of the device overlaps (or in most cases is inferior) with the time interval used for the collection and labeling of samples.

The liquid water based samples are processed by extraction with solid faze on the C18 extraction cartridge, while the organic once by extraction on $\mathrm{Si}$ cartridges. The solid samples are heated for the volatility of the interest chemical compounds that will be detected by IMS detection equipment, flame photometric detectors, indicating tubes or TENAX adsorbent resin tubes, which will under go GC/MS analysis.

For the functional testing, the liquid samples have been marked with DMMP, while the solids have been marked with HD. Before the testing, the chemical agents were analized, to check their purity.

The results of the testing of the prototipe corespond to the development specifications, under the principles of an integrated mechatronic device, i.e. a completely automated portable equipment for detection and identification of toxic compounds.

\section{GLOSSARY}

AP2C - A portable chemical contamination control mechatronic device, based on flame photometry, used to detect compounds of phosphorus (contained in G, V agents) and/or compounds of sulfur (contained in HD, V agents)

AP4C - A portable chemical contamination control mechatronic device, based on flame photometry, used to detect: all nerve agents, blood agents, blister agents and many TIC's and TIM's

CAM - Chemical Agent Monitor (is a portable mechatronic device based on IMS technology, capable of detecting nerve and blister agents)

CBRN - Chemical, Biological, Radiological and Nuclear

CWA - Chemical Warfare Agent

CWC - Chemical Weapons Convention

DMMP - Dimethyl methylphosphonate

FTIR - Fourier Transform Infrared Spectroscopy

GC/MS - Gas Chromatography coupled with Mass Spectrometry

HD - Yperite (Sulphure mustard)

IMS - Ionic Mass Spectrometry

IR - Infrared Spectrometry

L - - Lewisite

mg/l - Milligrams per litre

N - Nitrogen yperite (Nitrogen mustard)

NMR - Nuclear Magnetic Resonance

ppm - Parts per million

SPE - Solid Phase Extraction

SPME - Solid Phase Microextraction

TENAX - is a porous polymer resin based on 2.6-diphenylene oxide, used for the trapping of volatiles and semi-volatiles from air or which have been purged from liquid or solid sample matrices

TIC's - Toxic Industrial Compounds

TIM's - Toxic Industrial Materials 


\section{Acknowledgements}

This work was supported by a grant of the Romanian National Authority for Scientific Research and Innovation, CNCS/CCCDI - UEFISCDI, project number PN-III-P2-2.1-PTE-2016-0120, within PNCDI III.

\section{References}

[1] L.K. Lee, H. Brian. "Weapons of Mass Destruction, Detection". Encyclopedia of Espionage, Intelligence, and Security. 2004.

[2] D. Griffin. "Chemical Detection Equipment for Emergency Response. Military Technology". 30, 2006; no. 11, p. 54-58.

[3] R. Petre, T. Rotariu, T. Zecheru, N. Petrea, S. Băjenaru. „Environmental Long Term Impact on a Romanian Military Testing Range". Central European Journal of Energetic Materials, vol.13, 2016; no. 1, pp. 3-19.

[4] "STANAG 2103 NBC - NBC 4, Reporting nuclear detonations, biological and chemical attacks, and predicting and warning of associated hazards and hazard areas". Operators manual, (ATP-45 C),
(STANAG 2103, Edition 9) NATO/PfP UNCLASSIFIED. 2006.

[5] "Technical Manual for preparation and complete analysis of military interest chemicals from complex mixtures". Scientific Research Centre for NBC Defence and Ecology, $1^{\text {st }}$ ed., Bucharest. 2002.

[6] N. Petrea, V. Somoghi et all. "Device for Rapid Preparation of Samples for Detection and Identification of the Toxic Compounds". EEMJ, 2010; vol. 9 (1), p.51-55.

[7] S. Tony. "Briefing: NATO CBRN (Chemical, Biological, Radiological or Nuclear) Capabilities". Jane's Defense Weekly. March 5, 2008; 45, p.2426.

[8] "Rapid detection, alarm and monitoring device RAID M 100”. Operating Manual. 2007.

[9] AEP-10 - NATO, (2007), "Handbook for sampling and identification of biological and chemical agents (SIBCA). Procedures and Techniques". NATO Joint Capability Group (JCG) Chemical, Biological, Radiological and Nuclear (CBRN) Defence, SIBCRA Sub Group (AC/225), 1, Edition 5. 